\title{
Forward and Inverse Relational Models between Machined Surface Roughness and Key Parameters of Machining Process
}

\section{Ri Pan}

Beijing University of Technology

Ren Xingfei ( $D$ 19801252193@163.com )

Beijing University of Technology https://orcid.org/0000-0001-9986-0470

Zhenzhong Wang

Xiamen University

Dongju Chen

Beijing University of Technology

Jinwei Fan

Beijing University of Technology

\section{Original Article}

Keywords: Forward and inverse models, Surface roughness, Key parameters, Machining process, Magnetic composite fluid

Posted Date: March 17th, 2021

DOI: https://doi.org/10.21203/rs.3.rs-300729/v1

License: (c) (i) This work is licensed under a Creative Commons Attribution 4.0 International License. Read Full License 


\title{
Forward and Inverse Relational Models between Machined Surface Roughness and Key Parameters of Machining Process
}

Ri Pan ${ }^{1}$, Xingfei Ren ${ }^{1 *}$, Zhenzhong Wang ${ }^{2,3}$, Dongju Chen ${ }^{1}$, Jinwei Fan $^{1}$

${ }^{1}$ Key Laboratory of advanced manufacturing of Beijing, Beijing University of Technology, Beijing, 100124, China

${ }^{2}$ Department of Mechanical and Electrical Engineering, Xiamen University, Xiamen, 361005, China

${ }^{3}$ Shenzhen Research institute of Xiamen University, Shenzhen, 518000, China

*Correspondence: Xingfei Ren, Beijing University of Technology, Beijing, 100124, China

Email: 19801252193@163.com

\begin{abstract}
The relational model between machined surface roughness (MSR) and the adopted key machining parameters (KMPs) significantly influences the predictability and controllability of the machining process; therefore, it has attracted considerable attention. However, two critical problems still persist in this field. First, although most existing studies focus on the prediction model for MSR (forward model), wherein the MSR is dependent on input KMPs values, the inverse model that can calculate the KMP based on input MSR value is equally important; however, the inverse model has not been investigated as extensively as the forward model. The second issue is that most of the existing forward models are mainly established based on mechanism analysis; however, due to the complexity of most machining processes, the accuracy and generality of the model are not optimal. Therefore, this paper proposes a universal method for mathematically establishing the inverse model of the relation between the MSR and KMP. Initially, based on the response surface methodology, orthogonal experiments were designed and conducted, and the results were used to establish the forward model between the MSR and KMP. Subsequently, by combining the forward model with a self-developed genetic algorithm-based multiobjective optimization algorithm, an establishing method for inverse model between MSR and KMPs was proposed. Finally, experiments were conducted to validate the developed models. The experimental results show that for the forward model, all the 10 experimental MSR values approach the MSR values predicted by the forward model, and the average deviation was only approximately $7 \%$. Contrarily, for the inverse model, the average deviation was only approximately $7.64 \%$. Both these results verify the accuracy and effectiveness of the proposed models. With this method, as long as the desired processing results and constraints are given, the process parameters can be accurately derived.
\end{abstract}

Keywords: Forward and inverse models, Surface roughness, Key parameters, Machining process, Magnetic composite fluid 


\section{Introduction}

With the development of science and technology, the requirement of machining accuracy and efficiency of the components used in the high-end industries such as, aerospace and astronomy, and in the large laser devices, is increasing, resulting in a higher demand for the predictability and controllability of the machining process. An effective method to enhance the predictability and controllability is to establish an accurate relational model between the target variable and the adopted key machining parameters (KMP), and consequently, this has attracted much attention in various machining processes. Surface roughness after machining (that is, machined surface roughness, MSR) is an important target variable to be controlled in most machining methods, particularly precision machining processes. Therefore, the relational model between the MSR and KMP is widely studied.

Zhang et al. [1] established and verified a theoretical model to predict the machined surface roughness in an ultra-precision grinding process, considering the different removal mechanisms of the $\mathrm{Si}$ matrix and $\mathrm{SiC}$ particles. Wu et al. [2] proposed a prediction model for the surface roughness after grinding, considering the material ductile and brittle removal mechanisms, grain protrusion conditions, material properties, and machining parameters. Qi et al. [3] presented a prediction model of the surface roughness in belt polishing that comprehensively considered the characteristics of the polishing tool and workpiece. Lu et al. [4] established two comprehensive mathematical models to provide a better scientific understanding of the surface texture generation in the dual-axis wheel polishing (DAWP) process. The first model predicts the generation of micro-topography and roughness within a singleaxis tool influence function (TIF) area, based on the abrasive wear theory, abrasive distribution investigation, and accumulative removal theory. Based on this model, another surface microtopography generation model was also developed. Both models were validated through experiments. Saradhi et al. [5] developed an artificial intelligence-based model to understand the process mechanics and to predict the surface roughness and material removal rate (MRR) during laser-assisted turning of aluminum oxide using fuzzy logic. Zhang et al. [6] developed a novel theoretical roughness prediction model, into which the components of kinematics, plastic side flow, material elastic recovery, and cracks effect were integrated, to determine the underlying mechanisms of the surface roughness variation during oblique diamond turning of the potassium dihydrogen phosphate (KDP) crystal. Kong et al. [7] investigated improving the prediction accuracy of surface roughness in the milling process. Moreover, the authors developed a unique feature extraction method and comprehensively analyzed four types of Bayesian linear regression (BLR) models.

Based on the above literature, although establishing the relational model between MSR and KMP has attracted much attention in numerous machining methods, there are still two issues to be solved.

1. Most models are established based on mechanism analysis. However, because the mechanism of most machining processes is too complicated to be comprehensively understood [8], the model accuracy is affected. Hence, it is worthwhile to attempt mathematical modeling.

2. Most of the existing studies have focused on the forward model between the MSR and KMP (the forward model can be represented by Eq. (1)) that is used to predict the MSR using the provided KMP values.

$$
T=f\left(x_{1}, x_{2} \ldots \ldots x_{n}\right)
$$

where, $T$ is the MSR value, and $x_{1}, x_{2} \ldots \ldots x_{\mathrm{n}}$ are the KMPs. 
On the contrary, the inverse model between the MSR and KMP, represented by Eq. (2), has not been investigated as such. Nevertheless, the inverse relational model is equally important; it is used for determining the KMP values directly when the MSR value is provided. This is practical in the actual machining process. Therefore, the inverse model should also be studied.

$$
\left(x_{1}, x_{2} \ldots \ldots x_{\mathrm{n}}\right)=f^{-1}(\mathrm{~T})
$$

To solve the two aforementioned issues, this paper proposes a universal mathematical modeling method for the forward and inverse relational models between MSR and KMP. The proposed models are described in Section 2. Subsequently, in Section 3, the results of machining experiments are presented to validate the proposed method. Finally, the conclusions of this study are presented in Section 4.

\section{Universal establishing method for forward and inverse relational models between MSR and} KMP

This section consists of two parts: Section 2.1 presents the forward relational model between MSR and KMP using a mathematical method. Subsequently, the forward model is combined with a selfdeveloped genetic algorithm-based multi-objective optimization algorithm to develop the inverse model, as discussed in Section 2.2.

\subsection{Method for developing the forward model between MSR and KMP}

The method proposed for developing the forward model is a statistical method based on the response surface methodology (RSM). The proposed process is illustrated in Fig. 1.

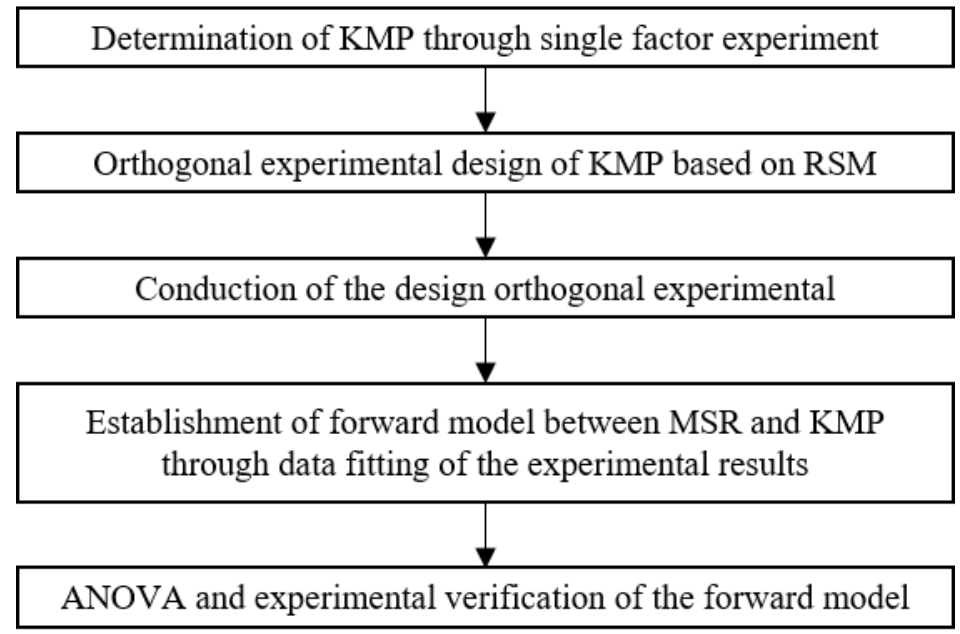

Figure 1. Method for developing the forward model between MSR and KMP.

(1) Determination of KMP by a single factor experiment

For a random machining process, a single factor experiment should be designed and conducted in advance to determine the KMPs, which have a significant impact on the MSR. In addition, the optimal value ranges of the KMPs should be determined in this step.

(2) Orthogonal experimental design and implementation based on RSM

The orthogonal experiments for the KMPs are designed based on the RSM and the optimal ranges of the KMPs. Thereafter, the experiments are conducted, and the experimental results (that is, the MSR values corresponding to the adopted KMPs) are used to construct the forward model.

(3) Establishment of the forward model between the MSR and KMP 
Based on the experimental results, the forward model between the MSR and KMP can be established according to RSM, which is essentially a multivariate quadratic regression equation fitting process. The general expression of the fitted equation is shown in Eq. (3).

$$
T=f\left(x_{1}, x_{2}, \ldots \ldots x_{n}\right)=\beta_{0}+\sum_{j=1}^{k} \beta_{j} x_{j}+\sum_{j=1}^{k} \beta_{j j} x_{j}^{2}+\sum_{i}^{k-1} \sum_{j}^{k} \beta_{i j} x_{i} x
$$

where $\beta_{i}(I=0, \ldots, k)$ are the regression coefficients.

(4) Validation of forward model

Because the forward model is developed by data fitting, first, the ANOVA of the forward model is conducted to validate the fitting effect. Subsequently, experiments were conducted to prove the correctness and effectiveness of the forward model.

The forward model between the MSR and KMP is established following the aforementioned steps; the model can predict the MSR if the KMP values are provided. This model is advantageous because it enhances the predictability of the machining process. However, it is not sufficient because in most of the actual machining processes, the MSR value is provided, and the values of KMP are required to be calculated for process control. The above situation cannot be addressed using the forward model, Eq. (3), because the number of unknowns is greater than that the equation can accommodate. Consequently, the inverse model between MSR and KMP, the expression of which is similar to Eq. (2), should be studied.

\subsection{Method for developing the inverse model between MSR and KMP}

It can be observed from Eq. (2) that the inverse model to be established is essentially related to the multi-objective optimization. With reference to the establishment of the kinematic and inverse kinematic models in the robot control field, the inverse model in this study is developed through a combination of the forward model and genetic algorithm (GA)-based multi-objective optimization algorithm.

From the mathematical perspective, the forward model, represented by Eq. (3), is essentially a spatial multidimensional surface, as shown in Fig. 2(a). It is obvious from Fig. 2(a) and (b) that, when the given MSR is the minimum value of the surface, only one group of KMPs can be found, that is, only one group of KMP can be used to obtain the minimum MSR using Eq. (3). On the contrary, when the provided MSR is not the minimum point, multiple groups of KMPs can satisfy Eq. (3).

Therefore, two types of situations should be considered when developing the inverse model between the MSR and KMP. 


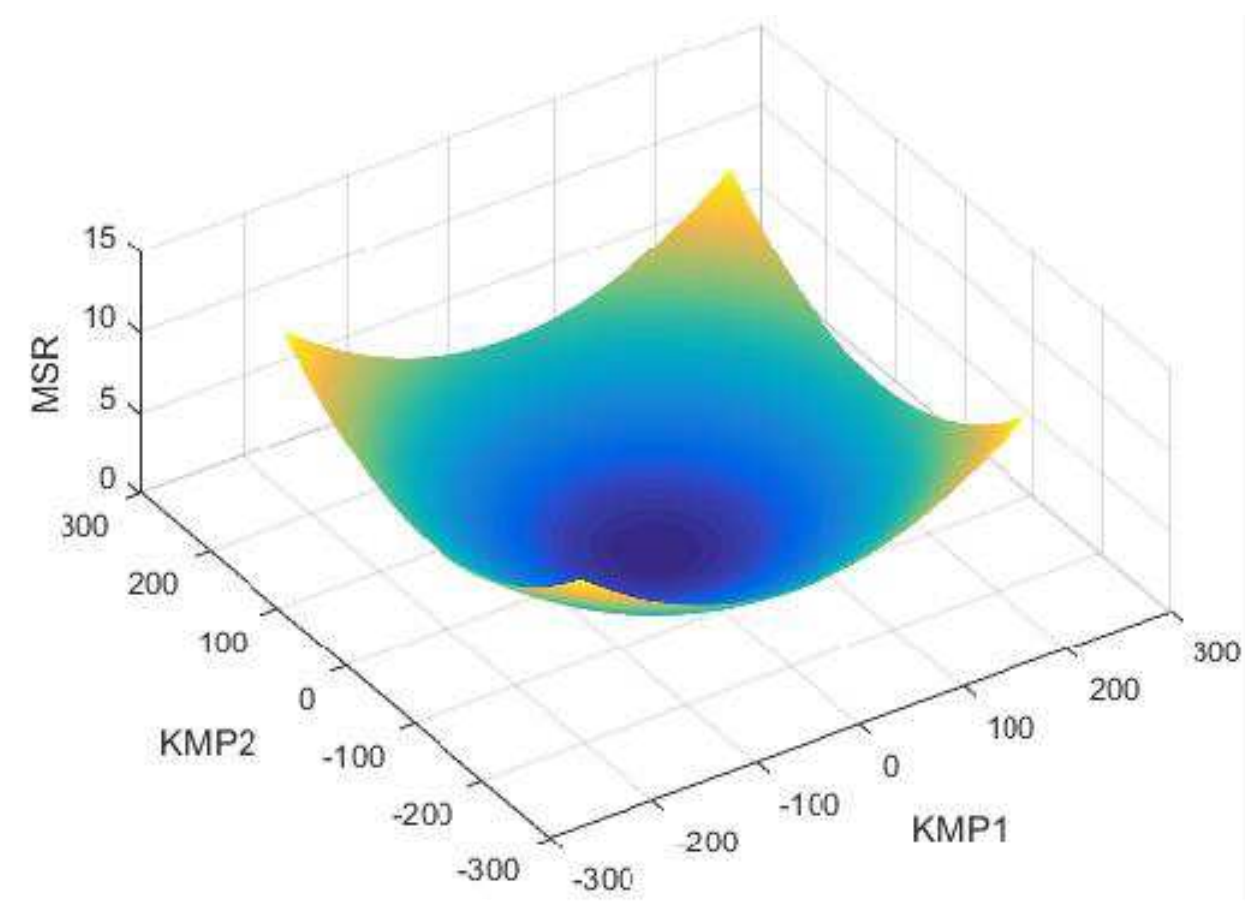

(a).

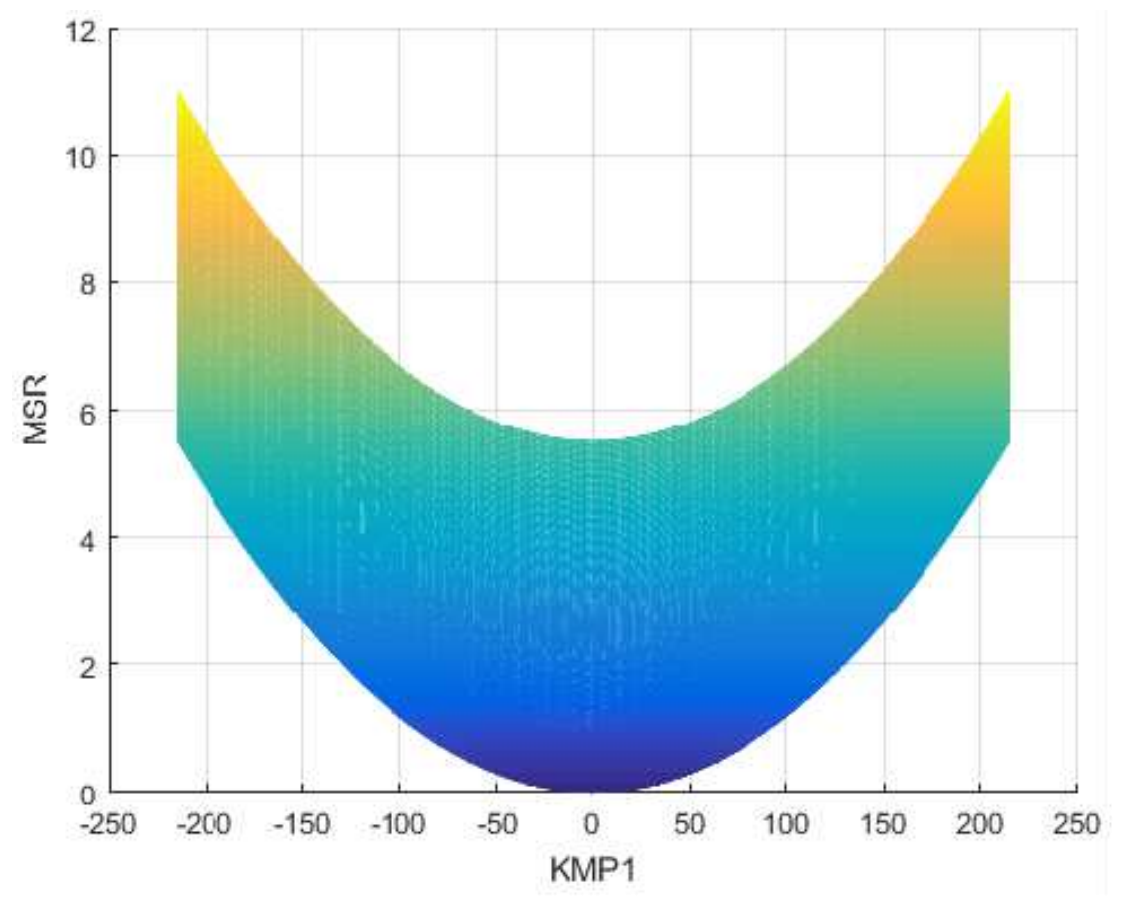

(b)

Figure 2. Spatial multidimensional surface.

(1) When the given MSR value is the minimum point of the forward model (that is, the spatial multidimensional surface):

Under such conditions, the GA can be used directly as the multi-objective optimization algorithm and can be combined with the forward model (Eq. (3)) to construct the inverse model. Fig. 3 shows the 
principle of the inverse model based on the GA and forward model, indicating the procedure according to which the KMPs are determined by the provided MSR, including the following steps.

(1) The parameters of GA were set, for example, the population size (for example, 100), crossover probability, mutation probability, and the initial and terminal iterative numbers. Subsequently, 100 groups of KMPs that were different from each other were randomly generated and used as the firstgeneration population.

(2)The 100 groups of KMPs were substituted into the forward model (Eq. (3)). Subsequently, 100 groups of MSR values were obtained and used to evaluate the fitness of each group of KMP.

(3) Based on the evaluation results, the top $5 \%$ groups of the first-generation population that had the best fitness were preserved, while the selection, crossover, mutation, and recombination operations were conducted on the other $95 \%$ groups to reform new groups. Subsequently, the preserved top $5 \%$ groups and the reformed $95 \%$ groups constituted a new generation population. Moreover, the following setting was applied: iterative number $=$ iterative number +1 .

(4) Step (2) is repeated until the terminal iterative number is reached. Finally, the group of KMPs with the best fitness (that is, minimum MRS) is provided as the output.

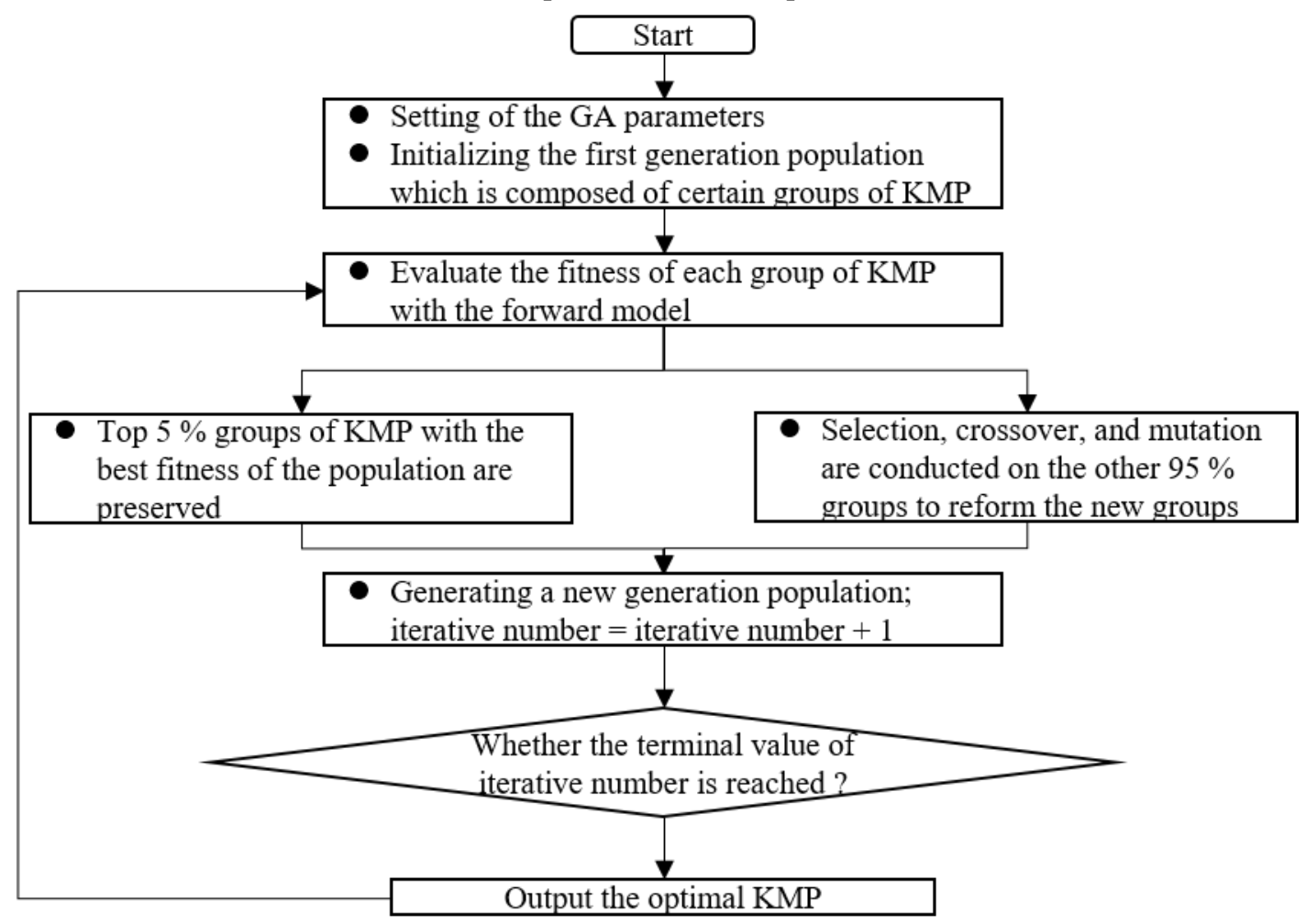

Figure 3. Principle of the inverse model based on GA and the forward model.

Based on the aforementioned process, the minimum MRS and its corresponding KMP can be obtained. The specific application of this part is explained in detail in the experimental section.

(2) When the given MSR value is a random value of the forward model:

As stated before, in this situation, the inverse calculation process of KMP is more complicated than the above situation; therefore, the fitness-evaluation function in the third step in Fig. 3 should be restructured.

First, the absolute of the difference between the given MSR value and the target variable expression is set as an optimization objective function, $G_{1}$, as shown in Eq. (4). 


$$
G_{1}=Y-T
$$

where $Y$ is the given MSR value, which is a constant. $T$ is the right-hand side of the forward model Eq. (3). Note that $G_{1}$ is a function that depends on the KMP.

Subsequently, the second optimization objective function $G_{2}$ depends on KMP is built, whose expression is similar to Eq. (3) as multivariate quadratic polynomials.

Finally, a comprehensive fitness-evaluation function $R$, is established as shown in Eq. (5). Here, $W_{1}$ and $W_{2}$ are constant coefficients.

$$
R=W_{1} G_{1}+W_{2} G_{2}
$$

After defining the fitness evaluating function $R$, the inverse calculation of KMP in this situation is also similar to the above situation, based on the process shown in Fig. 3, except for the variation of the fitness-evaluating function from the forward model, represented by Eq. (3), to Eq. (5). Accordingly, the inverse calculation of KMP using a provided MSR value can be achieved. The specific application of the algorithm will be explained in detail in the experimental verification phase.

Based on Section 2.1 and Section 2.2, the forward and inverse relational models of MRS and KMP can be achieved; the former is used to predict the MSR based on a provided KMP, and the latter is used to calculate the KMPs based on the provided MSR. The above two models are important for the predictability and controllability of the machining processes. The brass workpieces were polished using magnetic compound fluid (MCF) to verify the proposed method for developing the forward and inverse models.

\section{Experimental}

\subsection{Experimental details}

Fig. 4 (a) and (b) show the machining principle and structure of the MCF polishing machine employed in this study, respectively.

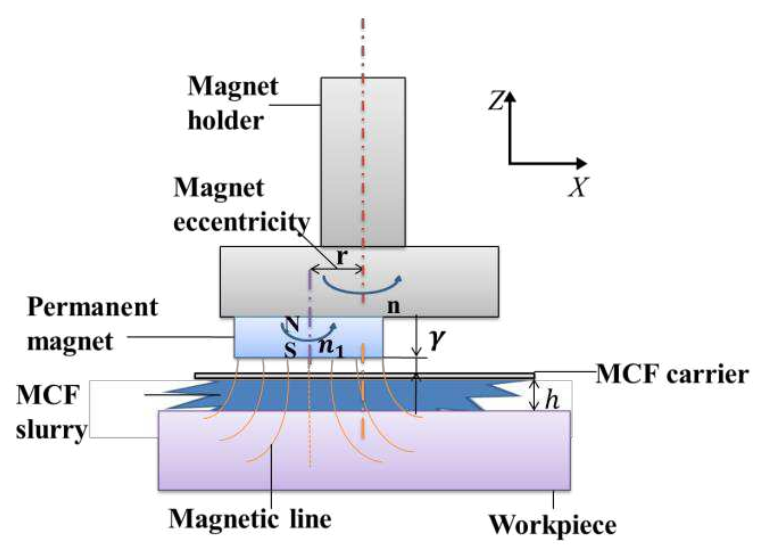

(a)

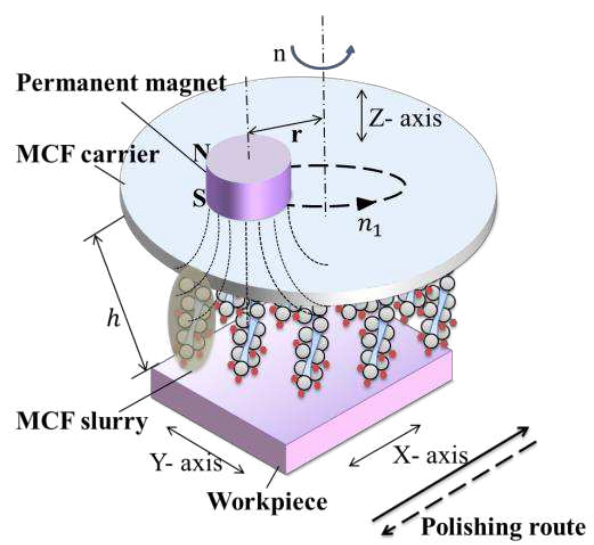

(b)

Figure 4. MCF polishing machine and principle employed in this study.

As shown in Fig 4(a), the permanent magnet is fixed below the magnet holder with an eccentric distance $r$ and is covered by the MCF carrier. Due to the permanent magnet field action, the viscous MCF slurry containing polishing abrasives changes from the fluid state to the Bingham medium and is adsorbed under the MCF carrier. Because the Bingham medium possesses a certain hardness, it can be used as a flexible polishing tool. 
To avoid negligible removal of the rotating center, the permanent magnet and the Bingham state MCF are driven simultaneously by two motors (Motor 1 and Motor 2 shown in Fig 4(a)). Fig 4(b) reveals the principle of the workpiece polished by the MCF. The rotating Bingham medium is driven simultaneously by Motor 1 and Motor 2 with rotational speeds of $n_{1}$ and $n$. When the Bingham medium is pressed toward the workpiece to a certain gap $h$, owing to the pressure and relative motion between the polishing tool and workpiece, the workpiece material is removed.

The other experimental conditions are as follows: The dimensions of the employed permanent magnet are $\varphi 40 \mathrm{~mm} \times \mathrm{t} 30 \mathrm{~mm}$. The magnetic field strength of the permanent magnet is approximately $0.4 \mathrm{~T}$, while the eccentricity $r=10 \mathrm{~mm}$. The dimensions of the MCF polishing carrier are $\varphi 120 \mathrm{~mm} \times$ $\mathrm{t} 3 \mathrm{~mm}$, and it is made of aluminum. In addition, the MCF used in this study was composed of magnetorheological fluid, carbonyl iron powder, $\alpha$-cellulose, and cerium oxide abrasive particles. The mass fractions of the above components are $28.0 \%, 61.7 \%, 3.3 \%$, and $7.0 \%$, respectively. The sizes of the cerium oxide abrasive particle and the carbonyl iron powder are 2-3 $\mu \mathrm{m}$ and $3-5 \mu \mathrm{m}$, respectively. The workpieces used in this study are copper H59, with dimensions are $50 \mathrm{~mm} \times 50 \mathrm{~mm}$ $\times 2 \mathrm{~mm}$. All the workpieces were ground before polishing, and the original roughness of the workpiece surface was approximately $0.2 \mu \mathrm{m}$.

Before and after polishing, the surface roughness of the workpiece was measured using a roughnessmeasuring instrument (modal TR300, manufactured by Time). During the measurement, eight points were uniformly selected on the workpiece surface, and the average value of the eight points was considered as the final measurement value. The measuring points before and after the test were the same.

\subsection{Experimental verification}

The experimental verification in this section is composed of two parts: development and verification of the forward model and development and verification of the inverse model.

\subsubsection{Establishment and verification of the forward model between MSR and KMP}

As shown in Fig. 1, the forward model between the MSR and KMP in MCF polishing is developed and validated through the following steps:

(1) Determination of KMP by a single factor

Based on our previous study on the MCF polishing process, the polishing effect is mainly dependent on the spindle speed ( $n$ in Fig 4(b)), polishing gap ( $h$ in Fig 4(b)), and polishing time $t$. Therefore, the aforementioned parameters are set as the KMPs. In addition, the optimal ranges of the machining parameters, $h, n$, and $t$ are 1-2 mm, 200-1000 rpm, and 30-120 min, respectively.

(2) Orthogonal experimental design and implementation based on RSM

Based on the KMPs and their optimal ranges, orthogonal experiments were designed according to the RSM, as stated in Section 2.1. A total of 17 groups of KMPs were obtained initially and were subsequently expanded to 61 groups without changing the basic principle.

Thereafter, the designed experiments for the 61 groups of KMPs were conducted. In particular, 61 brass workpieces with almost the same initial surface roughness were polished via MCF, and the MSRs were recorded, as shown in Table 1, to provide a basis for the next step of model development. 
Table 1. Designed experimental groups and the corresponding experimental results.

\begin{tabular}{ccccc}
\hline Sl. no. & $h(\mathrm{~mm})$ & $n(\mathrm{rpm})$ & $t(\mathrm{~min})$ & $\begin{array}{c}\text { Measured MSR } \\
(\mu \mathrm{m})\end{array}$ \\
\hline 1 & 1 & 200 & 30 & 0.102 \\
2 & 1 & 200 & 60 & 0.047 \\
3 & 1 & 200 & 75 & 0.044 \\
4 & 1 & 600 & 30 & 0.059 \\
5 & 1 & 600 & 30 & 0.092 \\
\hline 6 & 1 & 600 & 60 & 0.046 \\
7 & 1 & 600 & 90 & 0.046 \\
$\ldots \ldots$ & $\ldots \ldots$ & $\ldots \ldots . .$. & 0.088 \\
55 & 2 & 600 & $\ldots \ldots$ & 0.078 \\
56 & 2 & 600 & 90 & 0.109 \\
\hline 57 & 2 & 1000 & 30 & 0.078 \\
58 & 2 & 1000 & 60 & 0.079 \\
59 & 2 & 1000 & 75 & 0.079 \\
60 & 2 & 1000 & 90 & 0.078 \\
61 & 2 & 1000 & 120 & \\
\hline
\end{tabular}

(3) Development of the forward model between the MSR and KMP

According to Section 2.1(3), the values of KMP and the corresponding MSR shown in Table 1 were substituted into Eq. (3); after multivariate quadratic equation fitting, the regression coefficients in Eq. (3) were calculated. Therefore, the forward relational model between the MSR and KMPs of MCF polishing can be determined as shown in Eq. (6).

$$
\begin{aligned}
& M S R=\left(160703+97714.3 h+55.6943 n-1610.61 t+3.91987 h^{*} n-104.625 h * t\right. \\
& \left.+0.0812614 n * t+47414 h^{2}-0.0500214 n^{2}+8.02352 t^{2}\right) \times 10^{-6}
\end{aligned}
$$

(4) Verification of forward model

The verification of the forward model includes two parts: ANOVA and experimental verification.

(1) ANOVA of the forward model

Eq. (6) was analyzed via ANOVA to determine the significance of the impact of each machining parameter on the MSR and the prediction accuracy of the developed model. The analysis results are summarized in Table 2 . 
Table 2. ANOVA results of Eq. (6).

\begin{tabular}{|c|c|c|c|c|c|c|}
\hline Source & $\begin{array}{l}\text { Sum of } \\
\text { Squares }\end{array}$ & df & Mean Square & F-value & $p$-value Prob $>\mathrm{F}$ & \\
\hline Model & 0.0357 & 9 & 0.0040 & 36.1136 & $<0.0001$ & significant \\
\hline $\mathrm{h}$ & 0.0075 & 1 & 0.0075 & 68.4682 & $<0.0001$ & significant \\
\hline $\mathrm{n}$ & 0.0002 & 1 & 0.0002 & 1.6688 & 0.2022 & \\
\hline $\mathrm{t}$ & 0.0111 & 1 & 0.0111 & 100.8540 & $<0.0001$ & significant \\
\hline $\mathrm{h} \times \mathrm{n}$ & 0.0000 & 1 & 0.0000 & 0.0784 & 0.7807 & \\
\hline $\mathrm{h} \times \mathrm{t}$ & 0.0001 & 1 & 0.0001 & 0.4910 & 0.4867 & \\
\hline $\mathrm{n} \times \mathrm{t}$ & 0.0000 & 1 & 0.0000 & 0.1907 & 0.6642 & \\
\hline$h^{2}$ & 0.0018 & 1 & 0.0018 & 16.5893 & 0.0002 & \\
\hline$n^{2}$ & 0.0008 & 1 & 0.0008 & 7.5626 & 0.0082 & \\
\hline $\mathrm{t}^{2}$ & 0.0027 & 1 & 0.0027 & 24.9811 & $<0.0001$ & significant \\
\hline Residual & 0.0056 & 54 & 0.0001 & & & \\
\hline Total & 0.041 & 60 & & & & \\
\hline
\end{tabular}

Adequate precision $=21.824 ; \mathrm{R}^{2}=0.8644 ; \mathrm{R}^{2}$ (Adjusted) $=0.8404 ; \mathrm{R}^{2}$ (predicted) $=0.7816$

According to the ANOVA results in Table 2, the developed model has a high F-value, adequate precision, R-squared, adjusted R-squared, and predicted R-squared. All the above results indicate that the model can be used in a practical machining process.

(2) Experimental verification

To further verify the developed forward model, 10 groups of KMPs, listed in Table 3, were randomly generated. Note that all the 10 groups are different from the 61 groups of KMP used to develop the forward model. Subsequently, the 10 groups of experiments were conducted: 10 brass workpieces with the same initial surface roughness were polished by MCF, and the MSR of the polished surfaces were measured and compared with the predicted MSR with the forward model. The comparison results are listed in Table 3 and shown Fig. 5. 
Table 3. Comparison of the predicted and measured results.

\begin{tabular}{ccccccc}
\hline $\begin{array}{c}\text { Experimental } \\
\text { group }\end{array}$ & $g(\mathrm{~mm})$ & $n(\mathrm{rpm})$ & $t(\mathrm{~min})$ & $\begin{array}{c}\text { Deviation } \\
(\%)\end{array}$ & $\begin{array}{c}\text { Predicted } \\
\text { MSR }(\mu \mathrm{m})\end{array}$ & $\begin{array}{c}\text { Experimental } \\
\text { MSR }(\mu \mathrm{m})\end{array}$ \\
\hline 1 & 1.4 & 626 & 51 & 6.14 & 0.0694 & 0.0654 \\
2 & 1.4 & 481 & 106 & 7.51 & 0.0427 & 0.0398 \\
3 & 1.3 & 951 & 48 & -11.47 & 0.0647 & 0.0731 \\
4 & 1.5 & 901 & 48 & -3.84 & 0.0728 & 0.0758 \\
5 & 1.5 & 640 & 45 & 0.36 & 0.0788 & 0.0785 \\
6 & 1.8 & 698 & 50 & 6.32 & 0.0908 & 0.0854 \\
7 & 1.8 & 670 & 69 & 10.14 & 0.0759 & 0.0689 \\
8 & 1.6 & 366 & 58 & 3.36 & 0.0673 & 0.0651 \\
9 & 1.4 & 441 & 113 & 17.60 & 0.0420 & 0.0358 \\
10 & 1.8 & 577 & 69 & -4.07 & 0.0753 & 0.0785 \\
\hline
\end{tabular}

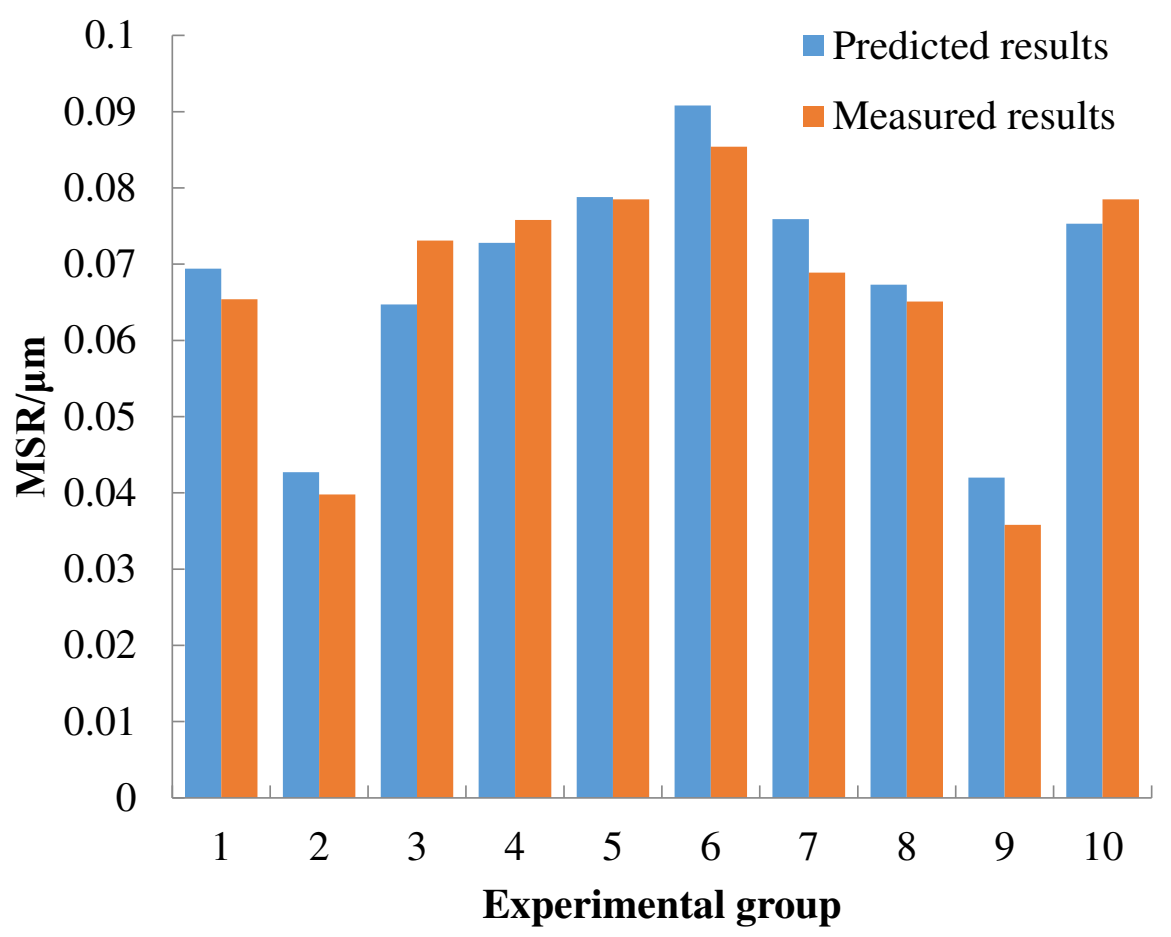

Figure 5. Comparison between the predicted and the measured results in the experimental verification of the forward model.

According to Table 3 and Fig 5, all the measured MSR values approach the predicted MSR. In particular, based on the 7 th row of Table 3, the maximum and minimum deviations between the predicted and the measured MSR are $17.60 \%$ and $0.36 \%$, respectively. Moreover, except for the maximum deviation, the deviations of most of the other experimental groups were $\leq \pm 10 \%$, and the average of absolute deviations was only approximately $7 \%$. Considering that the results are affected both by the precision of the prediction model and the various factors of the measurement error and the error due to the experimental environment, the predicted results are acceptable. The results support the correctness and effectiveness of the proposed forward model.

After developing and verifying the forward model, the inverse model is developed in the following section by the steps stated previously in Section 2.2. 


\subsubsection{Establishment and verification of inverse model between MSR and KMP}

According to Section 2.2, there are two types of inverse models based on the two types of the given MRS values.

(1) When the given MSR value is the minimum point of the forward model in Eq. (6):

In this situation, the inverse model is constructed based on a combination of the forward model (Eq. (6)) and GA. The principle of the inverse model is shown in Fig. 3. Therefore, when the required MSR is the minimum value of the forward model, the iterative calculation process in Fig. 3 is conducted to determine the corresponding KMP.

First, the parameters of GA, such as the population size, genetic algebra, crossover probability, and mutation probability, are defined, as listed in Table 4. Then, 100 groups of KMP are randomly generated within their optimal range of values and are defined as the first-generation population.

Table 4. Setting of the basic parameters of GA.

$\begin{array}{ll}\text { Size of population groups } & 100 \\ \text { Terminal value of iterative numbers } & 50 \\ \text { Possibility of cross production } & 0.7 \\ \text { Possibility of mutation } & 0.01\end{array}$

Subsequently, as shown in Fig. 3, the loop iterations are performed until the minimum MSR and its corresponding KMP values are determined. Fig. 6 shows the iterative calculation process for determining the minimum MSR and its corresponding machining parameters using the GA. The optimal MSR can be found through 20-25 iterations, whose value is $0.0295 \mu \mathrm{m}$, and the corresponding machining parameters, $h, n$, and $t$ are $1.1 \mathrm{~mm}, 200 \mathrm{rpm}$, and $106 \mathrm{~min}$, respectively.

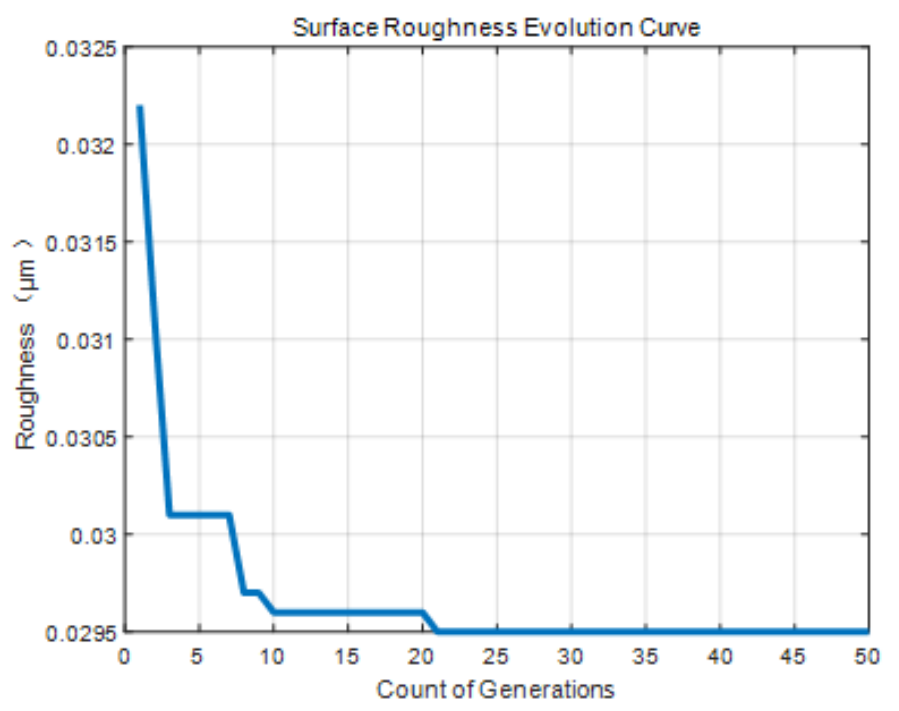

Figure 6. Iterative calculation process for determining the minimum MSR.

The correctness of the calculated result was verified through a polishing experiment of a brass workpiece based on the MCF, using the obtained KMP values, after which the MSR of the polished surface was measured and compared with the predicted MSR; the values are listed in Table 5.

Table 5. Comparison of the predicted and experimental results.

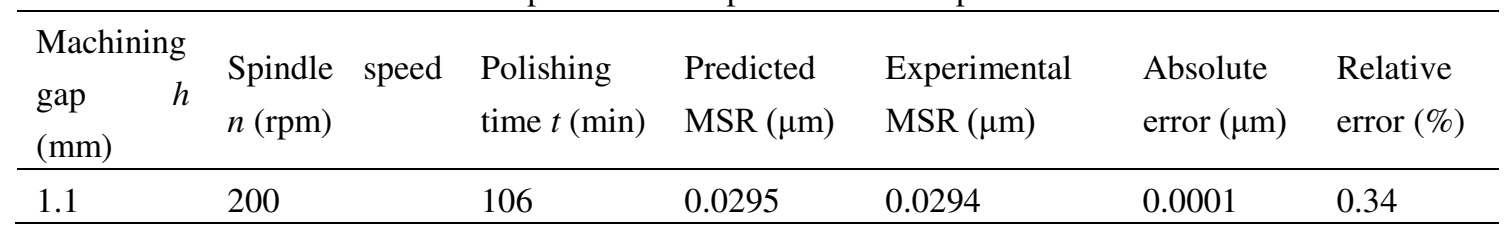


From Table 5, the optimal surface quality and optimal processing parameters obtained by the algorithm are highly consistent with the test results, with an absolute error of only $0.0001 \mu \mathrm{m}$ and a relative error of $0.34 \%$. The final surface roughness obtained was $0.0294 \mu \mathrm{m}$; this is smaller than any MSR in the previous experiments conducted in this study and listed in Table 1, indicating that the developed inverse model is effective.

(2) Development and variation of the inverse model with a randomly provided MSR

According to the establishment process of the inverse model in Section 2.2(2), in this situation, the KMPs are also calculated using the process shown in Fig. (3) in the above situation; the only difference is the fitness function used in the third step of Fig. (3) that should be restructured in the following steps.

(1) The optimization objective function $G_{1}$ is established as shown in Eq. (7).

$$
\begin{aligned}
& G_{1}=|\mathrm{Y}-M S R| \\
& M S R=(160703+97714.3 h+55.6943 n-1610.61 t+3.91987 h * n-104.625 h * t \\
& \left.+0.0812614 n * t+47414 h^{2}-0.0500214 n^{2}+8.02352 t^{2}\right) \times 10^{-6}
\end{aligned}
$$

where $\mathrm{Y}$ is the given MSR value, a constant; $G_{1}$ is a function depending on KMPs.

(2) The second optimization objective function $G_{2}$, which depends on KMP, is established as follows.

First, vector $P\left(h_{t}, n_{t}, t_{t}\right)$ is defined as the combination of the target KMPs, $h_{t}, n_{t}, t_{t}$ values, while vector $\mathrm{C}\left(h_{i}, n_{i}, t_{i}\right)$ is defined as the initial KMP values.

$G_{1}$ is defined as the Euclidean distance between $P$ and $C$, as expressed in Eq. (8), where $N$ is the normalization factor. This objective function for optimization ensures that the adjusted KMP values are as close as possible to the initial KMP values.

$$
G_{2}=\sqrt{((P-C) N)^{2}}
$$

In this example, vector $C$ is set as follows: $\mathrm{C}=\left[\begin{array}{l}1.5 \\ 60075\end{array}\right]$, where 1.5 is the median of the optimal range of the machining gap $h, 600$ is the median of the optimal range of the spindle speed $n$, and 75 is the median of the optimal range of the machining time $t$. In addition, the normalization factor $N$ is set as the inverse of the difference between the maximum and minimum values of the ranges of the machining parameters, $N=\left[\begin{array}{lll}1 & 1 / 800 & 1 / 90\end{array}\right]$.

Thereafter, the two optimization objectives $G_{1}$ and $G_{2}$ are combined to form a new function $R$, as shown in Eq. (9). In this study, the weight coefficients $W_{1}$ and $W_{2}$ are set as 100 and 1 , respectively, after several attempts, in advance.

$$
R=W_{1} G_{1}+W_{2} G_{2}
$$

Subsequently, $R$ is used as the fitness function of the GA-based multi-object optimization algorithm, as shown in the third step of Fig. 3. The iterative calculation shown in Fig. 3 can be conducted, and the KMP values satisfying the aforementioned optimization objectives can be determined.

Following the above steps, an inverse model between the MSR and the KMPs was established. Using this model, if the required MSR is provided, the KMP can be determined and provided to the machine operator. 
Three experimental groups were conducted to verify the above model, whose target MSRs were randomly set as $0.064 \mu \mathrm{m}, 0.057 \mu \mathrm{m}$, and $0.041 \mu \mathrm{m}$. Subsequently, using the inverse model, the KMPs were calculated to achieve the target MSR of the three experimental groups; they are listed in Table 6.

Table 6. Calculated KMPs to obtain the target MSR of the three experimental groups.

\begin{tabular}{ccccc}
\hline \multirow{2}{*}{ Experimental group } & \multirow{2}{*}{ Target MSR $(\mu \mathrm{m})$} & \multicolumn{3}{c}{ Calculated KMP by the inverse model } \\
\cline { 3 - 5 } & 0.064 & $h(\mathrm{~mm})$ & $n(\mathrm{rpm})$ & $t(\mathrm{~min})$ \\
\hline 1 & 0.057 & 1.5 & 594 & 62 \\
2 & 0.041 & 1.3 & 596 & 73 \\
3 & & 407 & 90 \\
\hline
\end{tabular}

Subsequently, three groups of MCF polishing experiments using the calculated KMP values were conducted. The initial surface roughness of all the brass workpieces was approximately $0.2 \mu \mathrm{m}$. After the experiments, the MSR was measured and compared with the target MSR. The results are listed in Table 7 and shown in Fig. 6.

Table 7. Comparison between the target MSR and the experimental MSR.

\begin{tabular}{cccccccc}
\hline $\begin{array}{c}\text { Experimental } \\
\text { group }\end{array}$ & $\begin{array}{c}h \\
(\mathrm{~mm})\end{array}$ & $\begin{array}{c}n \\
(\mathrm{rpm})\end{array}$ & $t(\mathrm{~min})$ & $\begin{array}{c}\text { Target } \\
\text { MSR } \\
(\mu \mathrm{m})\end{array}$ & $\begin{array}{c}\text { Experimental } \\
\text { MSR }(\mu \mathrm{m})\end{array}$ & $\begin{array}{c}\text { Absolute } \\
\text { error } \\
(\mu \mathrm{m})\end{array}$ & $\begin{array}{c}\text { Relative } \\
\text { error } \\
(\%)\end{array}$ \\
\hline 1 & 1.5 & 594 & 62 & 0.064 & 0.0565 & 0.0075 & 11.27 \\
2 & 1.5 & 596 & 73 & 0.057 & 0.0551 & 0.0019 & 3.33 \\
3 & 1.3 & 407 & 90 & 0.041 & 0.0375 & 0.0035 & 8.53 \\
\hline
\end{tabular}

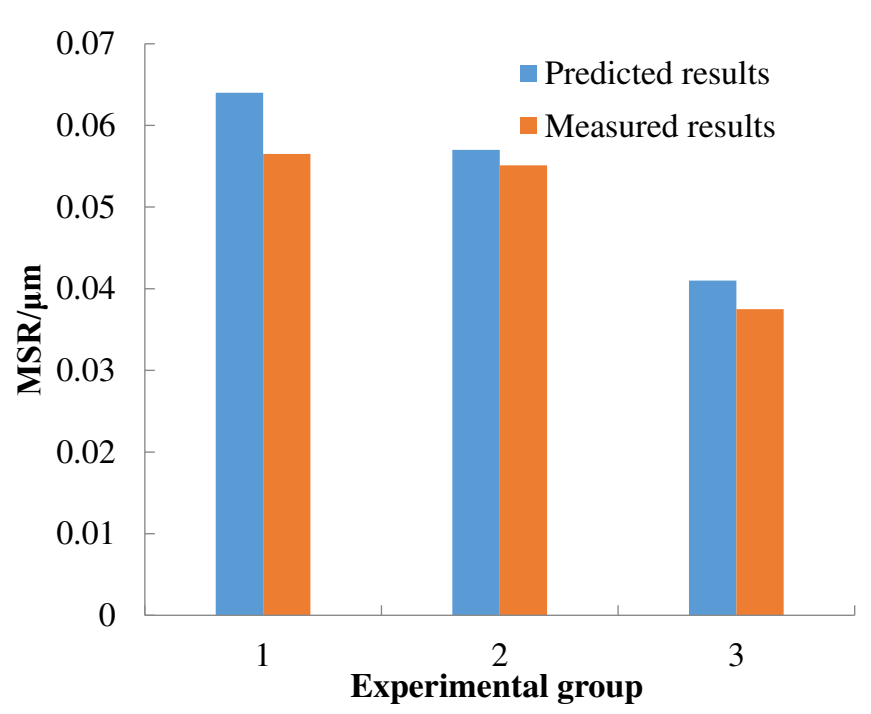

Figure 7. Comparison between the predicted and the measured results in the experimental verification of the inverse model.

It is obvious from Table 9 and Fig. 7 that by using the calculated KMP values in the inverse model, all the three experimental MSRs are close to the target MSR. In particular, the absolute errors between the experimental and target MRS of the three experimental groups are all less than $0.008 \mu \mathrm{m}$, and the relative errors are $11.27 \%, 3.33 \%$, and $8.33 \%$, respectively, while the average relative error is only $7.64 \%$. The results prove the precision and effectiveness of the inverse model. 
Based on the above results, it is also understandable that the inverse model can be used to calculate the KMPs when the required MSR is provided. This facilitates the precise control of the machining process.

\section{Conclusions}

To enhance the predictability and controllability of machining processes, a universal method for establishing forward and inverse models between MSR and KMPs was proposed in this study. The following conclusions are drawn.

1. The forward model between the MSR and KMP can be established based on the response surface methodology. The verification experiments of the forward model showed that all the 10 groups of the experimental MSR values were close to the MSR values predicted using the forward model, and the average deviation was only approximately $7 \%$; this support the prediction accuracy of the forward model, established through mathematical methods.

2. Two types of inverse models between MSR and KMP, related to the various types of the provided MSRs, were established based on combining the forward model and GA-based inverse calculation algorithm. The verification experiments of the inverse models have shown that for the first type of the inverse model, the deviation was only $0.34 \%$. On the contrary, the average deviation of the second inverse model was only $7.64 \%$. These results prove the correctness and effectiveness of the inverse models.

3. The forward model introduced in this paper can realize the accurate prediction of machining results, and the reverse model can realize the reverse calculation of the best machining parameters according to the given machining objectives.

\section{Availability of data and materials}

The datasets used or during the current study are available from the corresponding author on reasonable request.

\section{Competing Interests}

The authors declare that they have no competing interests.

\section{Funding}

This work was financially supported by the National Key Research and Development Project (Grant No. 2018YFB1107502), and the Science and technology project of Shenzhen (Grant No. JCYJ20180306172924636).

\section{Authors' Contributions}

Ri Pan was in charge of the whole trial; Xingfei Ren wrote the manuscript; Zhenzhong Wang assisted with experiment and verification; Jinwei Fan and Dongju Chen assisted with mathematical modeling and Programming. All authors read and approved the final manuscript.

\section{Acknowledgement}

We appreciate the invaluable expert comments and advices on the manuscript from all anonymous reviewers.

\section{References}


[1] ZHANG Z, YAO P, WANG J, et al. Nanomechanical characterization of RB-SiC ceramics based on nanoindentation and modelling of the ground surface roughness [J]. Ceramics International, 2020, 46(5): 6243-6253.

[2] WU J, CHENG J, GAO C, et al. Research on predicting model of surface roughness in small-scale grinding of brittle materials considering grinding tool topography [J]. International Journal of Mechanical Sciences, 2020, 166(2020):105263.

[3] QI J, ZHANG D, LI S, et al. Modeling and prediction of surface roughness in belt polishing based on artificial neural network [J]. Proceedings of the Institution of Mechanical Engineers, Part B: Journal of Engineering Manufacture, 2017, 232(12): 2154-63.

[4] LU A, JIN T, LIU Q, et al. Modeling and prediction of surface topography and surface roughness in dual-axis wheel polishing of optical glass [J]. International Journal of Machine Tools and Manufacture, 2019, 137(2019):13-29.

[5] PARDHA SARADHI V, SHASHANK V, SAI TEJA P, et al. Prediction of surface roughness and material removal rate in laser assisted turning of aluminium oxide using fuzzy logic [J]. Materials Today: Proceedings, 2018, 5(9): 20343-50.

[6] ZHANG S, ZONG W. A novel surface roughness model for potassium dihydrogen phosphate (KDP) crystal in oblique diamond turning [J]. International Journal of Mechanical Sciences, 2020, 173(2020): 105462.

[7] KONG D, ZHU J, DUAN C, et al. Bayesian linear regression for surface roughness prediction [J]. Mechanical Systems and Signal Processing, 2020, 142(2020): 106770

[8] PAN R, ZHONG B, CHEN D, et al. Modification of tool influence function of bonnet polishing based on interfacial friction coefficient [J]. International Journal of Machine Tools and Manufacture, 2018, 124(2018): 43-52. 
Figures

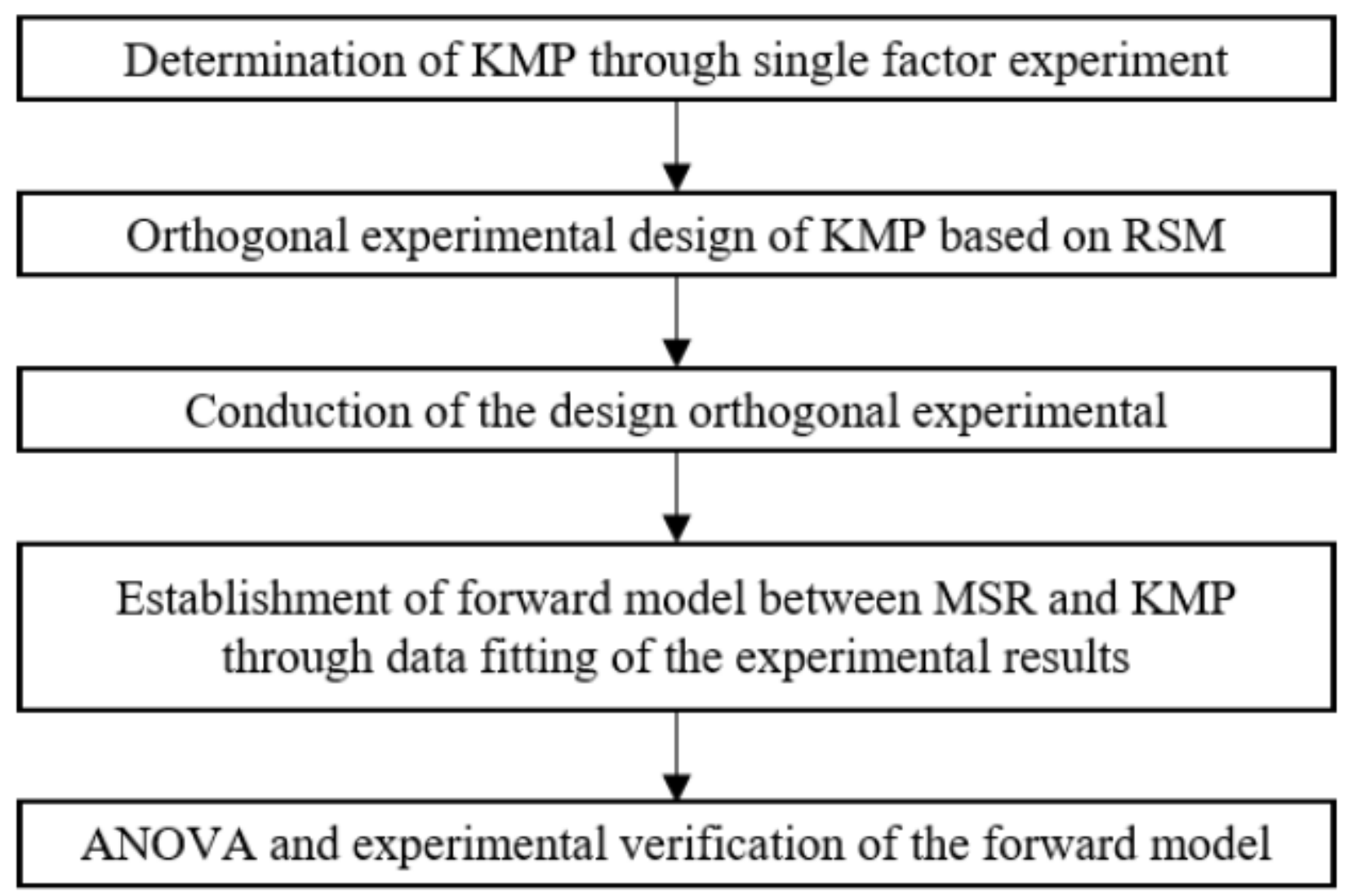

Figure 1

Method for developing the forward model between MSR and KMP. 


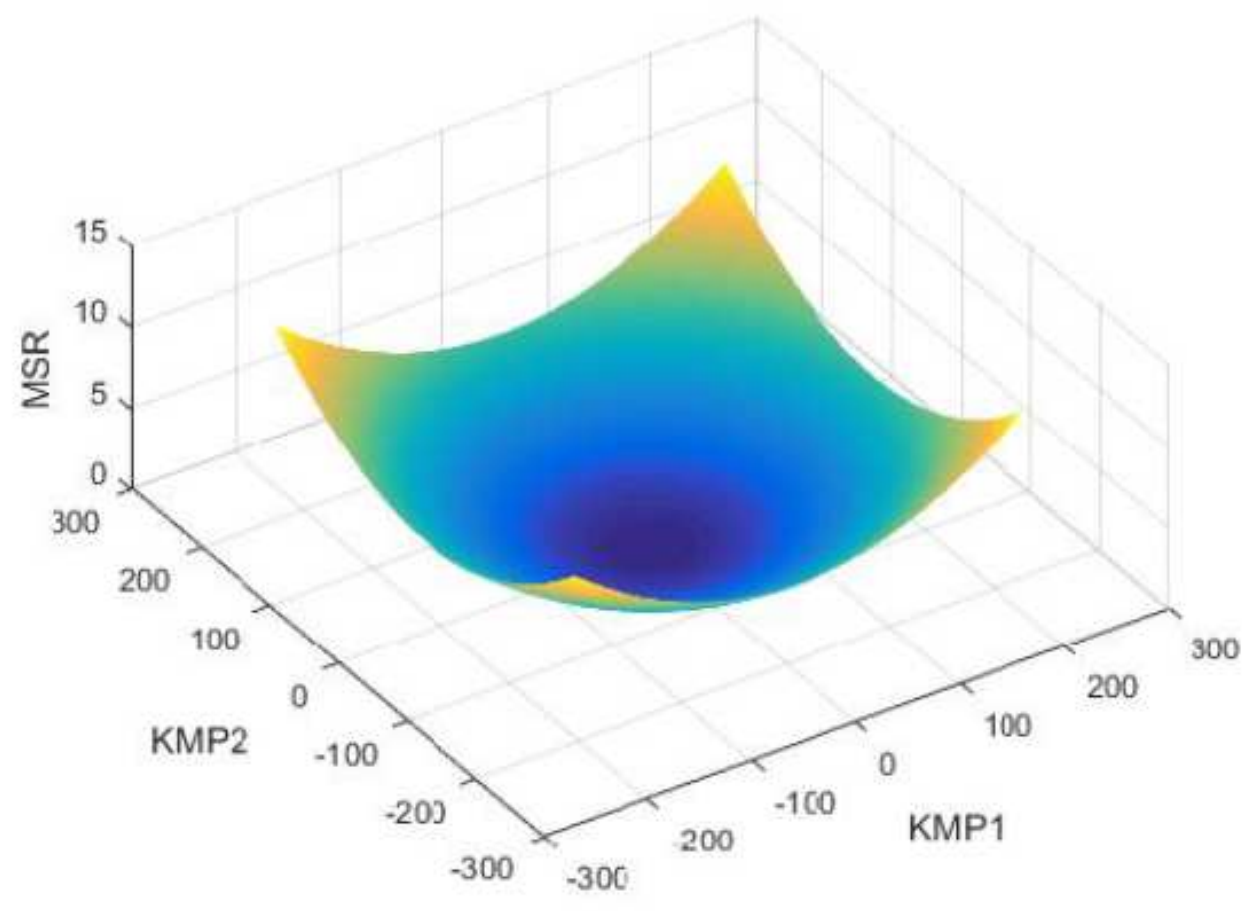

(a).

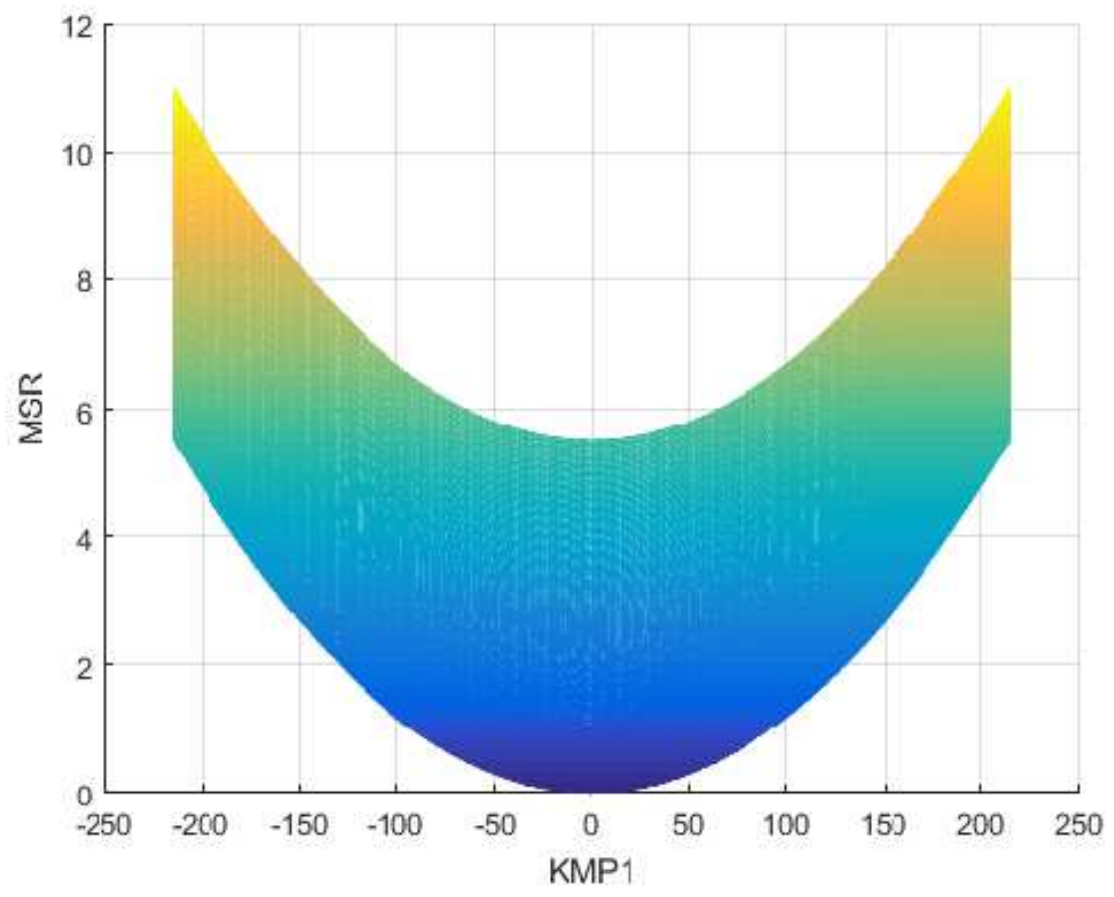

(b)

Figure 2

Spatial multidimensional surface. 


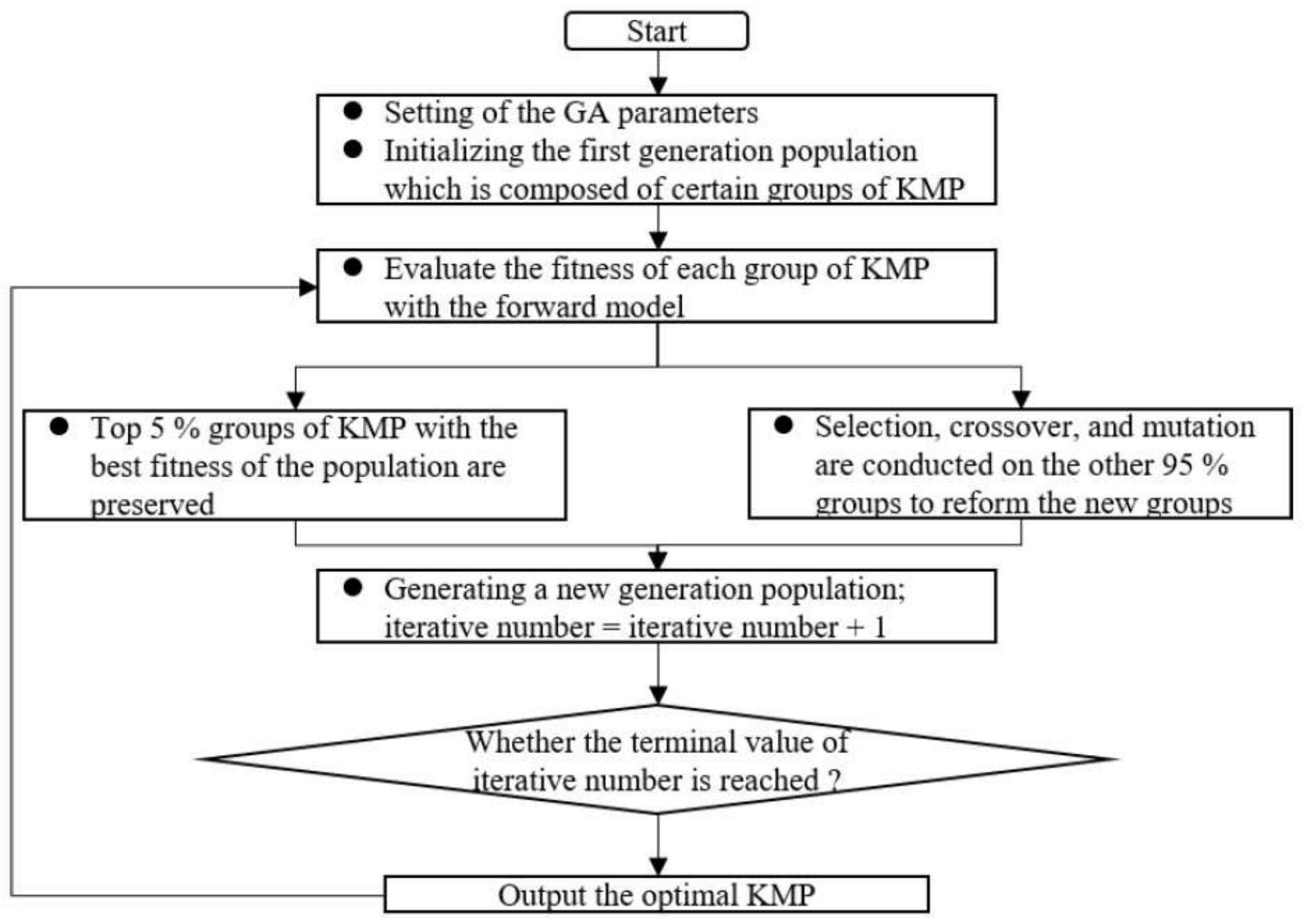

Figure 3

Principle of the inverse model based on GA and the forward model. 


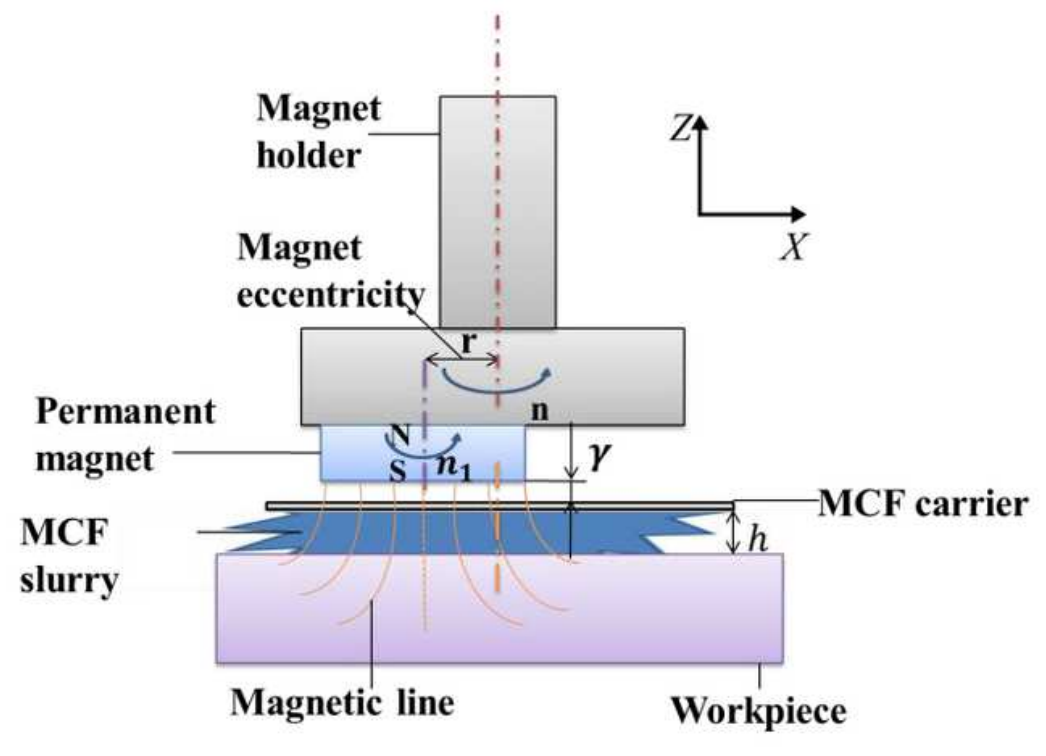

(a)

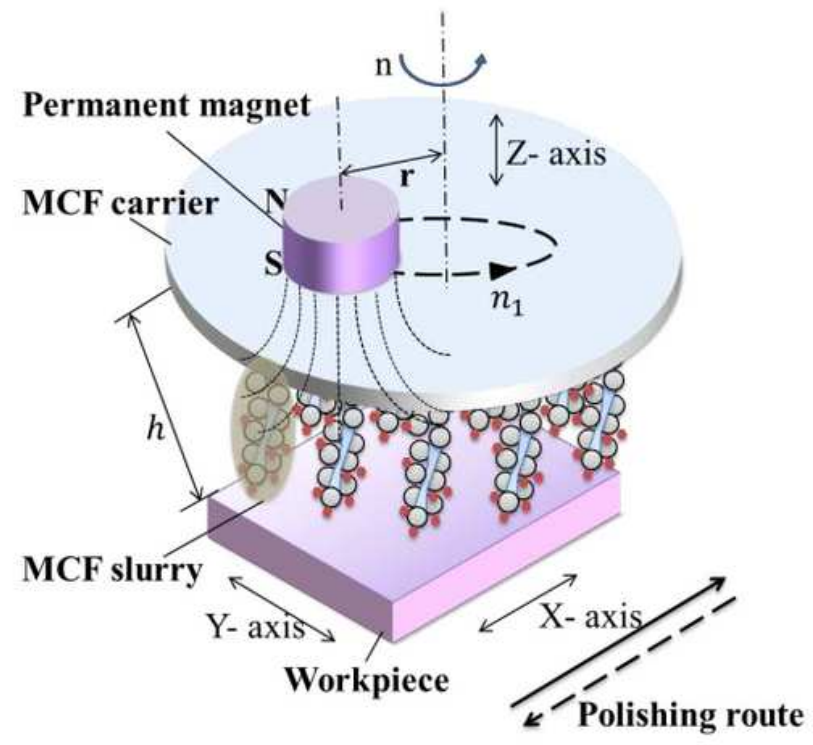

(b)

\section{Figure 4}

MCF polishing machine and principle employed in this study. 


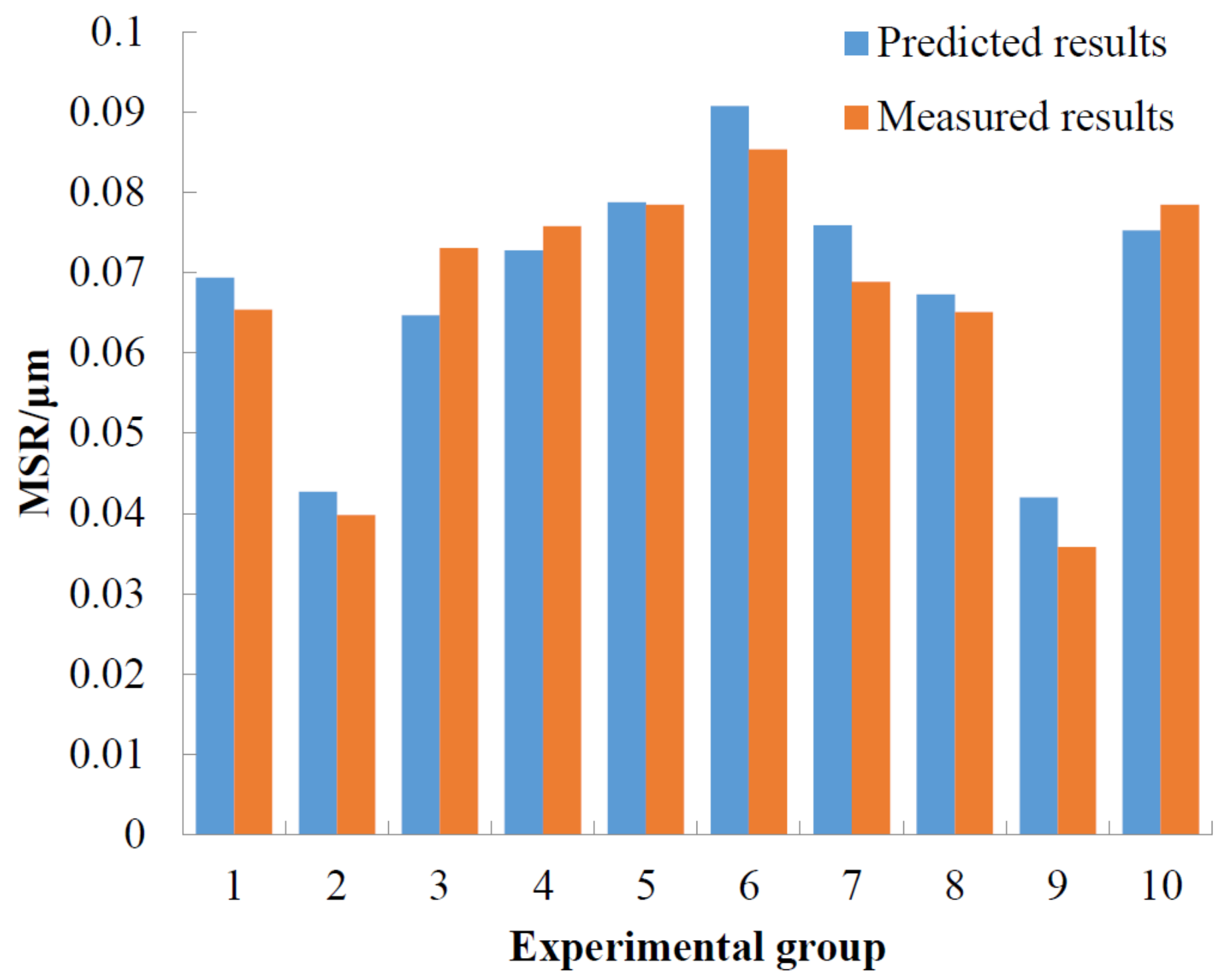

Figure 5

Comparison between the predicted and the measured results in the experimental verification of the forward model. 


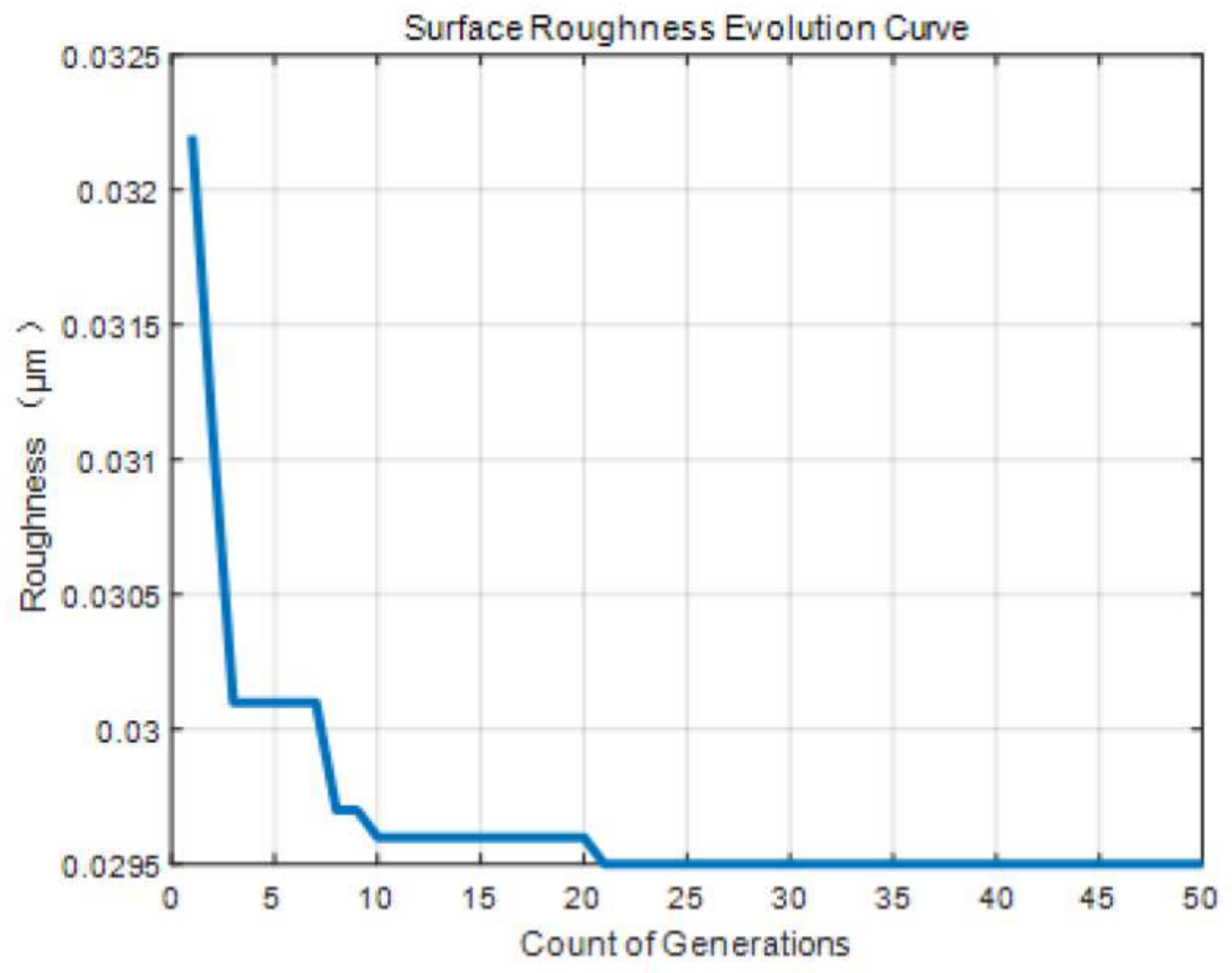

Figure 6

Iterative calculation process for determining the minimum MSR. 


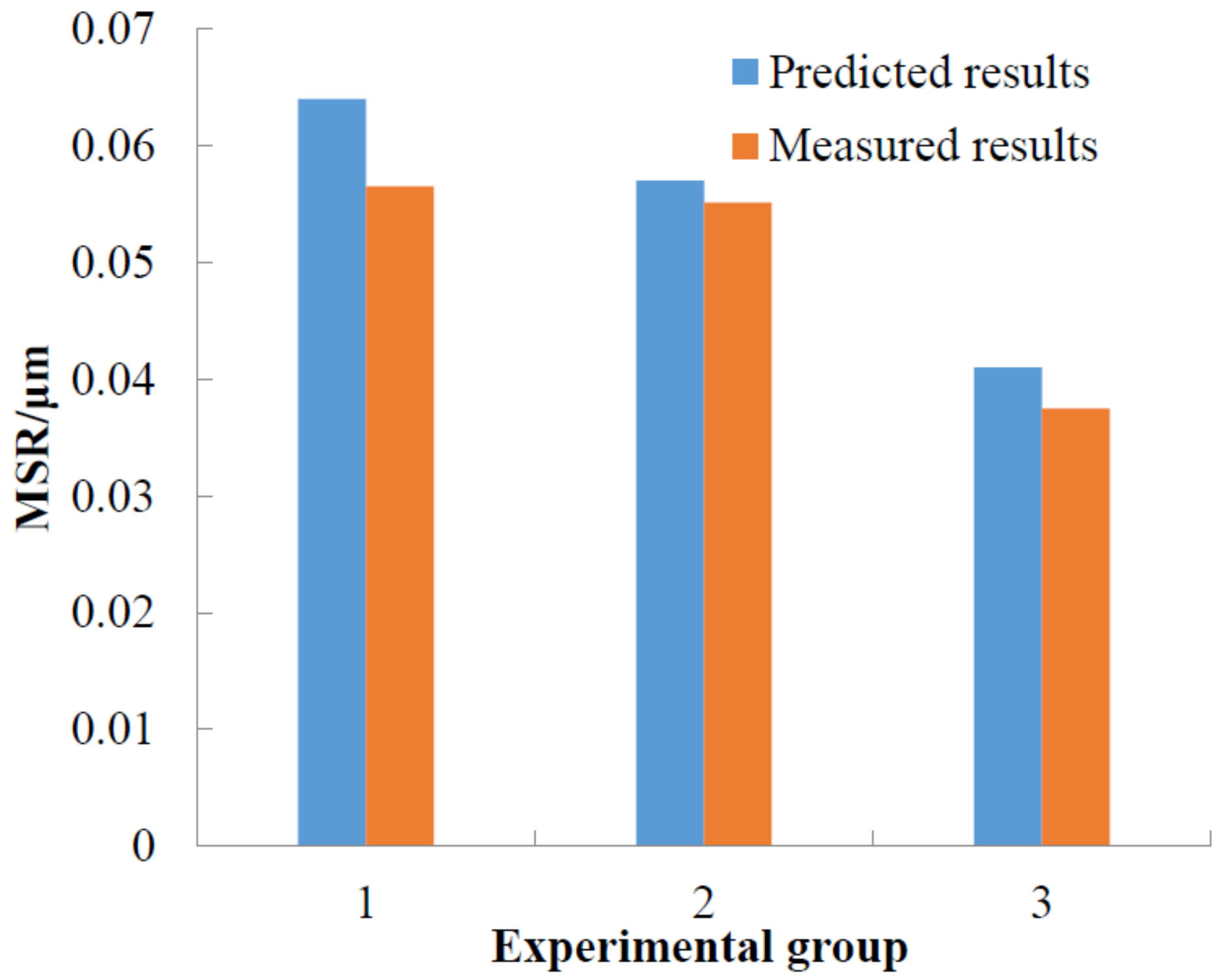

Figure 7

Comparison between the predicted and the measured results in the experimental verification of the inverse model. 